\title{
OCR using the Artificial Neural Network with Character Localization using Combined PCA and MSER Features
}

\author{
Sonu Rani \\ Department of CSE, \\ Punjabi University Regional Centre for Information \\ Information Technology and Management \\ Mohali, Punjab, India
}

\author{
Navpreet Kaur \\ Department of CSE, \\ Punjabi University Regional Centre for Information \\ Information Technology and Management \\ Mohali, Punjab, India
}

\begin{abstract}
The optical character recognition models are the algorithms to detect and extract the text from the image data. The optical character recognition (OCR) algorithms and the applications have gained the popularity among the IT researchers in the recent years. The OCR models have been utilized with the paper text scanning, natural scene text recognition, online sharing image tagging, etc. In this paper, the model has been proposed for the natural scene text recognition, which has been found the greater popularity over the years. The natural text recognition model has been found efficient in the case of automatic text recognition from the sign boards, LED boards and other such information collection from other visual sources. The proposed model specifically designed to work over the LED boards for the detection and extraction of the LED text, which is written in the combination of the small LED lights to form the shape of the character. The proposed model has been designed with the combination of the shape recognition, text region detection and character recognition method using the maximally stable extremal regions (MSER), principal component analysis (PCA) and the artificial neural networks (ANN). The proposed model has been designed as the robust text region detection and localization solution along with the text recognition method. The proposed model has undergone the various experiments for the evaluation of the performance from the various aspects altogether. The proposed solution has performed as per the expectation and outperformed the existing model in the terms of detection and recognition accuracy.
\end{abstract}

\section{Keywords}

Text detection, MSER, ANN, PCA, Text localization

\section{INTRODUCTION}

Preliminary step for automatic text recognition is text detection . It should be speedy, efficient and vital for feed an OCR classifier with the st input. Or we can say that, segmented regions must correspond to the actual text. Due to its vast applicative techinque - from querying text in grahic content for signboard translation using PDA - text detection has been given a lot of observation across the last decade and even earlier. As opposed to the traditional text along with black ink on a white paper, images of natural or inevitable scenes introduce difficulties that are away from being completely solved, like uneven illumination, complex backgrounds, complex signs that can be simply misclassified as text,complex text styles and perspective projections.

Now days, more and more databases are multimedia in nature of the development of the digital technology. The databases usually consist images and videos to the textual information. The textual information is very helpful semantic information because it explain the image or video and can be used to fully understand. Text localization can be done in three types of images which are following below.

\section{Document image}

2. Scene or graphic text image

3. Caption text image

Document images may have struture of CD covers, scanned book covers or video images. Text in videos or images are also known as scene text and caption text. Scene text is also known as graphics text. Natural images that consist of text or character are called scene text. Artificial text is type of caption text and text or words are added or superimposed in the image by this one only [5]. There are two various approaches have been used for text localization from difficult images namely region based approach and texture based approach

Many text detection technique have been developed on, mathematical morphology, spatial-frequency image analysis, binarization and edge detection [1]. Usually text detection technique can be grouped as either connected-component based, edge-based and texture-based methods [2]. In a manner [1] By edgebased text detection the better performance were attained. It acquire top overall performance among 4 methods including color-based character extraction and mathematical morphology. Edge-based text detection has also been used in alliances to edge profiles. Park et al. [2, 3 the automatic detection and recognition of Korean text use in outdoor signboard images. However, they suppose that a distinct text sign is located about the middle line of the image.

\section{LITERATURE REVIEW}

Jo, Kanghyun et. al. [1] has proposed the LED Dot matrix text recognition method in natural scene. This paper proposes a technique for LED text detection and recognition in natural scene images. To execute this aim of detection and recognition of a character and text, it include of two main steps with the following steps: the first step: the Canny edge was help to detect character pixels which appear in LED display area from scene images.

Yin, Xu-Cheng et. al. [2] has worked on the robust text detection in natural scene images. A fast and effective pruning algorithm is designed to extract in this paper. Maximally Stable External Regions (MSERs) techinque as character candidates using the schedule of minimizing regularized variations.

Iqbal, Khalid et. al. [3] has proposed Bayesian network scores based text localization in scene images. In this paper, Bayesian network scores using K2 algorithm in conjunction with the geometric features based effective text localization 
method with the help of maximally stable external regions (MSERs). To find text regions, all MSER-Situated extracted candidate characters are directly correlate to an existing text or line localization method. Second, adjacent extracted MSER-situated candidate characters are not encompassed into text or line regions due to rigid edges constraint. So, By use of selection rules extracted candidate character regions are incorporated into text regions.

Gupta, Neha et. al. [4] has worked on the localization method for text localization in Complex Images Using Haar Wavelet Transform. By this paper, a new hybrid approach is developed which locate text in different backareas. However, discrepancy of text due to differences in alignment, size, orientation, style, and as well as low image contrast and complex background create the issue of automatic text localization highest challenging.

Mishra, Anand et. al. [5] has implemented a top-down and bottom-up cues for scene text recognition algorithm. In this paper, the authors have concentrate on the issue of recognizing text extracted from street or road images. The authors have prompt the framework that exploits both topdown and bottom-up cues. The bottom-up cues are derived from individual character detections from the image.

Ikica, Andrej [6] has worked on the improvement of edge profile based method for text detection in images of natural scenes. The internet search engines to the PDA signboard translators in many applicative areas is the Understanding of visual text content is of a vital importance. Images of natural scenes, however, pose numerous difficulties compared to the traditional scanned documents.

\section{EXPERIMENTAL DESIGN}

The proposed model begins with the acquisition of the LED board image. Then the proposed model performs the various kinds of preprocessing methods to normalize the lighting effects and color ingredient. Afterwards the image undergoes canny edge detection and contour selection methods in the combination. The canny edge detection and contour selection select the character shapes and return the coordinates of the character. Edge detection algorithm has been deployed for the character location detection, which further undergoes the neural network classification using the probabilistic neural network algorithm for the purpose of character reconstruction on each extracted character. Then the PCA method is used for the purpose of matching the character images with the character templates. The matching characters will be finalized and the text is formed after combining the character to make the word and words to the lines.

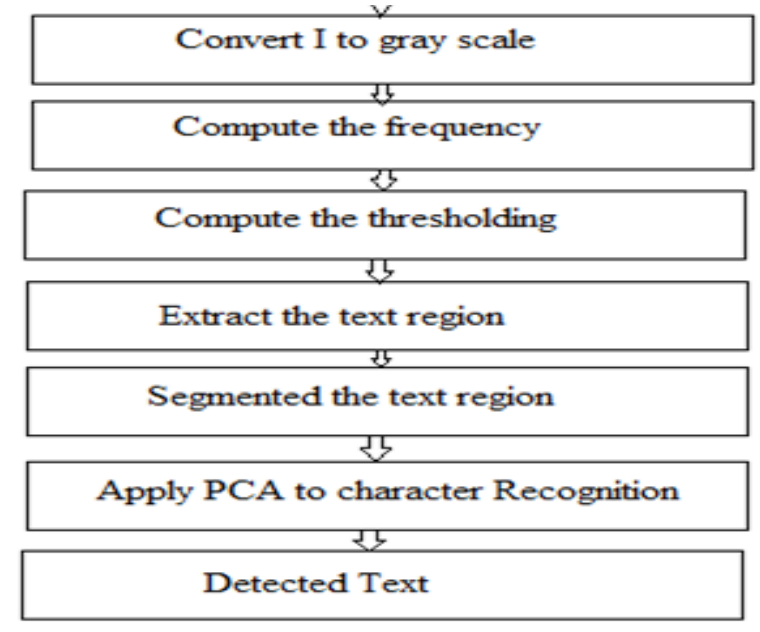

Figure1: The projected working of the proposed model

Algorithm 1: Text Region Localization and Character Recognition Algorithm

1. Image selection dialogue

2. Acquire the image file and convert it to the matrix

3. Check the input image and select the appropriate options (Multiple option selection is enabled)
a. Blurring
b. Flipping or,
c. Denoise

4. Process the image with the options set on the step 3

5. Make the region selection

6. Perform the binarization over the selected region

7. Apply the MSER over the binarized image

8. Extract the regions containing the text

9. Count the regions and iterate for each of the region

a. Perform the character level detection

b. Perform the character edge detection

c. Extract the character region from the detected text region

d. Apply the PCA over each of the extracted character

e. Load the training data

f. Load the group matrix

g. Restructure the PCA matrix and prepare the test data

h. Set the essential neural network parameters

i. Apply the neural network over the test and training data

j. Return the matching character

$\mathrm{k}$. If it's the last character

1. Return the iteration

m. Otherwise

n. GOTO 9(a).

10. Get all of the characters with the POS tagging

11. Arrange the characters according to lines

12. Return the recognized text

\section{RESULT ANALYSIS}

In this research project, the work has been performed using the proposed model for the character recognition in the natural scene. The natural scene text extraction procedure has used several morphological methods amalgamated with the principle component analysis for character recognition. The text region is extracted using the proposed model's centrally weighted morphological operations. The natural scene text 
recognition can assist the vehicle drivers, and make their ride easy and informative to read all of the traffic messages flashed on the road. This can provide the critical information to the drivers and hence can assist them to avoid the traffic hours, road blockage due to accidental hazards, etc.

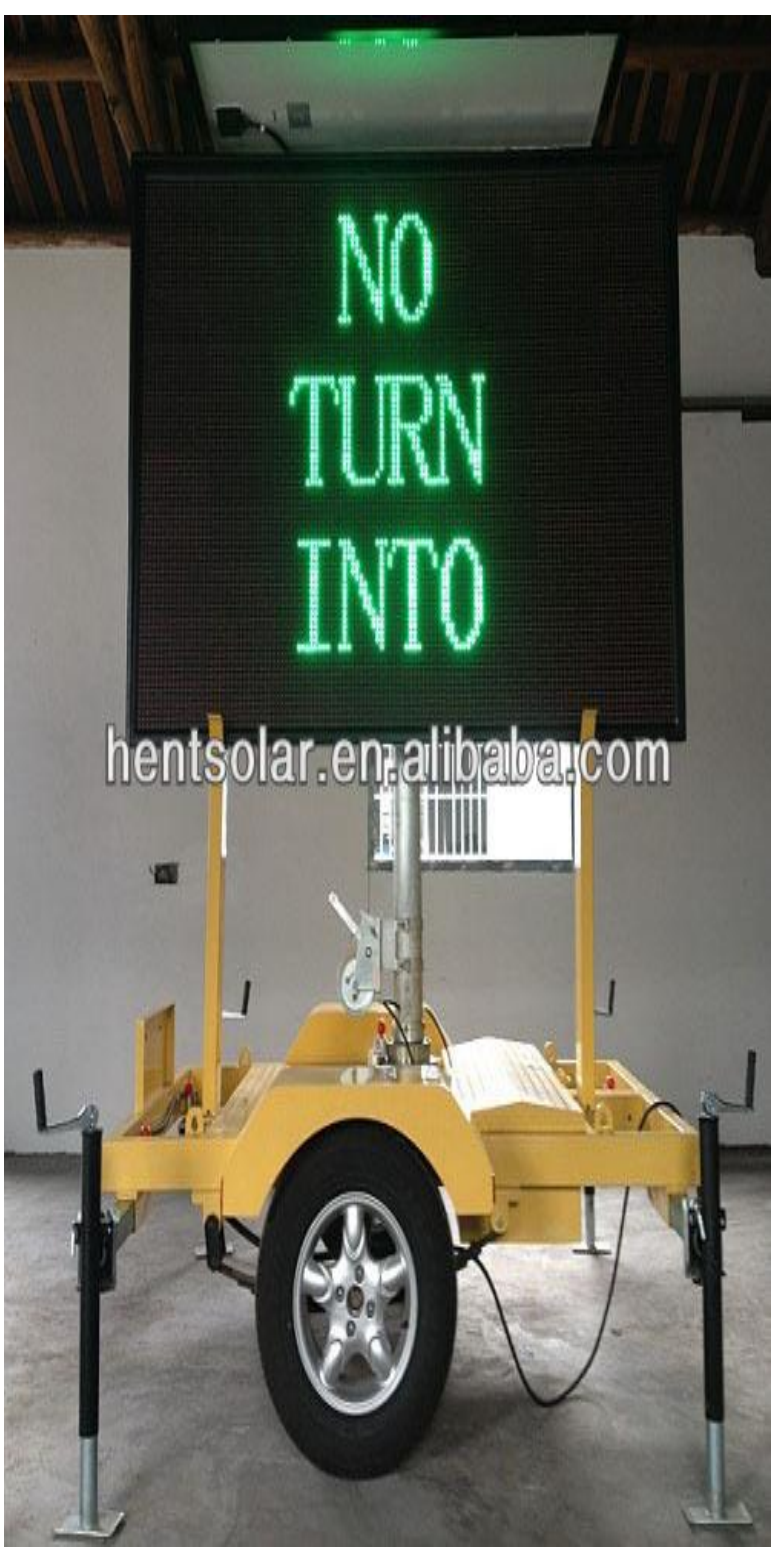

Figure 2: The figure from the dataset for led text recognition

We have changed over the picture into grayscale from its hued particular. At that point we have utilized middle channel to de-clamor the auto picture in the event of any commotion created in the picture securing procedure. Subsequently, we have utilized picture widen morphological strategy to improve the splendor of the picture to highlight the number plate district. Later, we have utilized article sketching out determination strategy with the end goal of extraction of the different protests in the picture. In the last step, we have utilized the number plate extraction area utilizing the most noticeable articles chose in the recent step and utilizing it with littler article evacuation strategy for morphological picture handling.

The proposed calculation is intended to give back the slipped by time per revolution and aggregate reaction time for all pivots. The precision of the project is figured on the premise of sort 1 and 2 factual mistakes. Sort $1 \& 2$ factual slips incorporate the genuine positive, genuine negative, false positive and false negative qualities. On the premise of the recent parameters, specificity and affectability are likewise figured.

Table 1: Showing performance of proposed system on per sample basis.

\begin{tabular}{|c|c|c|c|}
\hline $\begin{array}{c}\text { File } \\
\text { Index }\end{array}$ & $\begin{array}{c}\text { Total } \\
\text { Characters }\end{array}$ & $\begin{array}{c}\text { Correctly } \\
\text { Detected } \\
\text { Characters }\end{array}$ & $\begin{array}{c}\text { Character Level } \\
\text { Accuracy in } \\
\text { Percentage } \\
\end{array}$ \\
\hline 1 & 4 & 4 & 100 \\
\hline 2 & 4 & 4 & 100 \\
\hline 3 & 5 & 0 & 0 \\
\hline 4 & 5 & 5 & 100 \\
\hline 5 & 5 & 4 & 80 \\
\hline 6 & 8 & 8 & 100 \\
\hline 7 & 8 & 5 & 62.5 \\
\hline 8 & 9 & 4 & 44.44444444 \\
\hline 9 & 9 & 9 & 100 \\
\hline 10 & 10 & 10 & 100 \\
\hline 11 & 10 & 10 & 100 \\
\hline 12 & 10 & 0 & 0 \\
\hline 13 & 12 & 12 & 100 \\
\hline 14 & 13 & 8 & 61.53 \\
\hline 15 & 13 & 13 & 100 \\
\hline 16 & 15 & 15 & 100 \\
\hline 17 & 16 & 10 & 62.5 \\
\hline 18 & 16 & 12 & 75 \\
\hline 19 & 19 & 18 & 94.73684211 \\
\hline 20 & 38 & 29 & 76.31578947 \\
\hline \multicolumn{3}{|c|}{ AVERAGE ACCURACY } & 77.851354 \\
\hline
\end{tabular}

\section{CONCLUSION}

The proposed model has been based upon the artificial neural network (ANN) based classification along with the robust feature descriptors for the detection and localization of the text regions. The proposed model has been designed to utilize the principal component analysis (PCA) along with the maximally stable extremal regions (MSER) extraction. The extremal regions are primarily utilized for the informative information region extraction, which further undergoes the character location determination in order to extract the individual character candidates out of the image containing the text. The proposed model has been tested with total 20 images, where the proposed model has been considered sufficiently accurate. The overall accuracy has been improved from $73 \%$ to $77 \%$ when grossly evaluated for the overall results based upon the number of the correctly classified characters. 


\section{REFERENCES}

[1] Gupta, Neha, and V. K. Banga. "Localization of Text in Complex Images Using Haar Wavelet Transform." International Journal of Innovative Technology and Exploring Engineering (IJITEE) 1 (2012): 111.

[2] Ikica, Andrej, and Peter Peer. "An improved edge profile based method for text detection in images of natural scenes." In EUROCON-International Conference on Computer as a Tool (EUROCON), 2011 IEEE, pp. 1-4. IEEE, 2011.

[3] Iqbal, Khalid, Xu-Cheng Yin, Hong-Wei Hao, Sohail Asghar, and Hazrat Ali. "Bayesian network scores based text localization in scene images." In Neural Networks (IJCNN), 2014 International Joint Conference on, pp. 2218-2225. IEEE, 2014.
[4] Jo, Kanghyun. "LED Dot matrix text recognition method in natural scene", Neuro computing, vol 1, issue 1, pp. 1033-1041, Science Direct (Elsevier), 2015.

[5] Kaur, Tajinder, and Nirvair Neeru. "Text Detection and Recognition from Natural Scene", vol 4, no. 7, pp. 32113216, IJARCET 2015.

[6] Kaur, Tajinder, and Nirvair Neeru. "Text Extraction from Natural Scene using PCA", vol 5, issue 7, pp. 272-277, IJCSET 2015.

[7] Mishra, Anand, Karteek Alahari, and C. V. Jawahar. "Top-down and bottom-up cues for scene text recognition." In Computer Vision and Pattern Recognition (CVPR), 2012 IEEE Conference on, pp. 2687-2694. IEEE, 2012.

[8] Yin, Xu-Cheng, Xuwang Yin, Kaizhu Huang, and HongWei Hao. "Robust text detection in natural scene images." Pattern Analysis and Machine Intelligence, IEEE Transactions on 36, no. 5 (2014): 970-983. 